PROC. OF JSCE,

NO. 204, AUG. 1972

\title{
SIMULATION OF MULTI-CORRELATED RANDOM PROCESSES AND APPLICATION TO STRUCTURAL VIBRATION PROBLEMS*
}

\author{
By Masaru HoSHIYA**
}

\section{INTRODUCTION}

This paper presents a practical simulation method of a set of $m$ multi-correlated stationary Gaussian processes, $f_{i} c(t), i=1,2, \cdots, m$ with zero mean for a prescribed cross-spectral density matrix. The processes can be simulated by using a trigonometric series with Gaussian random coefficients;

$$
\begin{aligned}
f_{i}(t)= & \sum_{p=1}^{i} \sum_{k=1}^{N}\left[a_{i p}(k) \cos \left\{\omega_{k} t-\alpha_{i p}(k)\right\}\right. \\
& \left.+b_{i p}(k) \sin \left\{\omega_{k} t-\alpha_{i p}(k)\right\}\right] \\
& i=1,2, \cdots, m
\end{aligned}
$$

The engineering applications are numerous. Only a few of these are also presented.

Although many authors dealt with simulation of a single random process utilizing trigonometric series ${ }^{10), 5)}$, filtered white noise ${ }^{1)}$ and correlated random pulse trains ${ }^{9)}$ etc., only Shinozuka ${ }^{12}$ ) and Shinozuka and Jan ${ }^{13)}$ and Borgman ${ }^{3)}$ studied simulation of multi-correlated processes.

The essential feature of Shinozuka and Jan's approach is to employ a mathematical model of a series of cosine functions by

$$
\begin{aligned}
f_{i}(t)= & \sum_{p=1}^{i} \sum_{k=1}^{N}\left|H_{i p}\left(\omega_{k}\right)\right| \sqrt{2 \Delta \omega} \\
& \times \cos \left(\omega_{k}{ }^{\prime} t+\theta_{i p}\left(\omega_{k}\right)+\varphi_{p k}\right)
\end{aligned}
$$

in which the weighted coefficients $\left|H_{i p}\left(\omega_{k}\right)\right| \sqrt{2 \Delta \omega}$ associated with the specified cross spectral density matrix are deterministic and almost evenly spaced frequencies $\omega_{k}{ }^{\prime}$ are random. $\varphi_{p k}$ are also uni-

* This is a revised manuscript of the previous two papers

(1) Simulation of Multi-Correlated Random Processes and

(2) Simulation of Structural Vibration, submitted to JSCE dated November and December, 1971 respectively.

** Ph.D., Assistant Professor, Musashi Institute of Technology, Civil Engineering Department, Tokyo formly distributed random variables.

Instead, the mathematical model eq. (1) involves only one set of randomized coefficients $a_{i p}(k)$ and $b_{i p}(k)$ and the method is simple and practical.

\section{MULTI-CORRELATED STATIONARY PROCESSES}

The simulation of $f_{i}^{c}(t)$ by eq. (1) can be attained with the following conditions;

(a) $a_{i p}(k)$ and $b_{i p}(k)$ for every $i, p$ and $k$ are independent but identically distributed Gaussian variables with zero mean.

(b) $a_{i p}(k)$ and $a_{j p}(k)$, therefore also $b_{i p}(k)$ and $b_{j p}(k)$ for fixed $p$ and $k$ are statistically correlated with covariance,

where

$$
K_{i j p}(k)=2\left|H_{i p}\left(\omega_{k}\right)\right|\left|H_{j p}\left(\omega_{k}\right)\right| \Delta \omega
$$

$$
\Delta \omega=\left(\omega_{u}-\omega_{l}\right) / N \text { and } \omega_{k}=\omega_{l}+(k-1 / 2) \Delta \omega
$$

$\omega_{u}$ and $\omega_{l}$ are respectively the upper and lower cutoff frequencies in the positive range of the spectrum which has significant intensity. For the prescribed cross-spectral density matrix, $\boldsymbol{S}^{c}(\omega)$ of the element $S_{i j}{ }^{c}(\omega)$ which is the cross-spectral density function of $i$ th and $j$ th processes, $H_{i p}\left(\omega_{k}\right)$ can be given by

$$
\begin{aligned}
\boldsymbol{S}^{c}\left(\omega_{k}\right)=\boldsymbol{H}\left(\omega_{k}\right) \overline{\boldsymbol{H}}^{T}\left(\omega_{k}\right) \\
= \\
\left(\begin{array}{r}
H_{11}\left(\omega_{k}\right) \\
H_{21}\left(\omega_{k}\right), H_{22}\left(\omega_{k}\right) \\
H_{31}\left(\omega_{k}\right), H_{32}\left(\omega_{k}\right), H_{33}\left(\omega_{k}\right) \\
\vdots \\
\vdots \\
H_{m 1}\left(\omega_{k}\right), H_{m 2}\left(\omega_{k}\right), H_{m 3}\left(\omega_{k}\right), \cdots, H_{m m}\left(\omega_{k}\right)
\end{array}\right) \times \\
\left(\begin{array}{r}
\bar{H}_{11}\left(\omega_{k}\right), \bar{H}_{21}\left(\omega_{k}\right), \cdots \cdots \cdots \cdots, \bar{H}_{m 1}\left(\omega_{k}\right) \\
\bar{H}_{22}\left(\omega_{k}\right), \cdots \cdots \cdots \cdots, \bar{H}_{m 2}\left(\omega_{k}\right) \\
\bar{H}_{33}\left(\omega_{k}\right), \cdots, \bar{H}_{m 3}\left(\omega_{k}\right) \\
\vdots \\
\ddots \\
\bar{H}_{m m}\left(\omega_{k}\right)
\end{array}\right)
\end{aligned}
$$


where $\overline{\boldsymbol{H}}^{T}\left(\omega_{k}\right)$ is the transpose of the complex conjugate of the lower triangular matrix. Since $\boldsymbol{S}^{c}\left(\omega_{k}\right)$ is hermitian and non-negative but not necessarily positive definite, the above decomposition of $\boldsymbol{S}^{c}\left(\omega_{k}\right)$ is not always possible. The method of solution for $H_{i p}\left(\omega_{k}\right)$ with the special case described above is given in the reference ${ }^{13)}$ and will not be repeated here.

(c) Except for the case (b), $a_{i p}(k)$ and $b_{i p}(k)$ for every $i, p$ and $k$ are statistically independent. (d) $\alpha_{i p}(k)$ is the phase angle and is given by

$$
\alpha_{i p}(k)=\tan ^{-1}\left\{\frac{\operatorname{Im} H_{i p}\left(\omega_{k}\right)}{\operatorname{Re} H_{i p}\left(\omega_{k}\right)}\right\}
$$

It is easy to show that the ensemble average of $f_{i}(t)$ is zero. The cross correlation function of $f_{i}(t)$ and $f_{j}(t+\tau)$ is with the aid of conditions (a), (b) and (c);

$$
\begin{aligned}
R_{i j}(\tau) & =E\left\{f_{i}(t) f_{j}(t+\tau)\right\} \\
& =\sum_{p=1}^{i} \sum_{k=1}^{N} K_{i j p}(k) \cos \left\{\omega_{k} \tau-\alpha_{j p}(k)+\alpha_{i p}(k)\right\}
\end{aligned}
$$

This indicates that the process $f_{i}(t)$ is stationary. The expression $f_{i}(t)$ of eq. (1) is a linear sum of a set of independent random variables $a_{i p}(k)$ and $b_{i p}(k)$ with normal distribution and give rise to another random variables with a normal distribution function. Consequently, $f_{i}(t)$ represents a stationary Gaussian process with zero mean.

The cross-spectral density function of $f_{i}(t)$ and $f_{j}(t)$ is by virtue of the Wiener-Khintchine relations

$$
S_{i j}(\omega)=\frac{1}{2 \pi} \int_{-\infty}^{\infty} R_{i j}(\tau) e^{-i \omega \tau} d \tau
$$

Upon substitution of eq. (7), together with eqs. (3) and (6), it becomes;

For $\omega \geqq 0$,

$$
S_{i j}(\omega)=\sum_{k=1}^{N} \sum_{p=1}^{i} H_{i p}\left(\omega_{k}\right) \bar{H}_{j p}\left(\omega_{k}\right) \Delta \omega \delta\left(\omega-\omega_{k}\right)
$$

Thus, $\lim _{N \rightarrow \infty} S_{i j}(\omega)=\sum_{p=1}^{i} H_{i p}(\omega) \bar{H}_{j p}(\omega)=S_{i j} c(\omega)$

For $\omega<0$,

$$
S_{i j}(\omega)=\sum_{k=1}^{N} \sum_{p=1}^{i} \bar{H}_{i p}\left(\omega_{k}\right) H_{j p}\left(\omega_{k}\right) \Delta \omega \delta\left(\omega+\omega_{k}\right)
$$

Thus, $\lim _{N \rightarrow \infty} S_{i j}(\omega)=\sum_{p=1}^{i} \bar{H}_{i p}(-\omega) H_{j p}(-\omega)$

$$
=\sum_{p=1}^{i} H_{i p}(\omega) \bar{H}_{j p}(\omega)=S_{i j} c(\omega)
$$

Equations (8) and (9) indicate that $f_{i}(t)$ becomes the target process $f_{i}^{c}(t)$ when $N$ is infinity. For practical purpose of simulation, large $N$ can be used.

Consider the generation procedure of $a_{i p}(k)$ and $b_{i p}(k)$ of eq. (1). First construct the following random vector with zero mean for fixed $k$ and $p$.

$$
\begin{array}{r}
A_{p k}=\left\{a_{1 p}(k), a_{2 p}(k), \cdots, a_{2 p}(k),\right. \\
\left.\cdots, a_{j p}(k), \cdots, a_{m p}(k)\right\}
\end{array}
$$

Since the elements $a_{i p}(k)$ are Gaussian with zero mean and $i$ th and $j$ th elements $a_{i p}(k)$ and $a_{j p}(k)$ are statistically correlated with the covariance $K_{i j p}(k)$, they are jointly Gaussianly distributed. If $i=j, K_{i j p}(k)$ becomes the variance. Since the direct trial of simulation of $A_{p k}$ is very complicated because of the jointly correlated property, first sample $m$ independent Gaussianly distributed variables $\zeta_{p k}=\left\{\zeta_{1 p}(k), \zeta_{2 p}(k), \cdots\right.$, $\left.\zeta_{m p}(k)\right\}$ with zero mean and unit variance by a Monte Carlo random sampling technique ${ }^{8)}$. These are then subjected to a linear transformation $\boldsymbol{C}_{p k}$, after which the resulting random vector $A_{p k}$ will have the specified matrix of covariances $\boldsymbol{K}_{p k}$, whose elements are $K_{i j p}(k)$. It is noted that the property of Gaussian distribution of $A_{p k}$ is preserved under the linear transformation.

In what follows, the determination of $\boldsymbol{C}_{p k}$ is suggested in such a manner that it transforms $\zeta_{p k}$ to $A_{p k}$ with the specified matrix $\boldsymbol{K}_{p k^{11}}$.

Consider a lower triangular matrix $C_{p k}$ as

$$
\boldsymbol{C}_{p k}=\left(\begin{array}{l}
C_{11 p}(k) \\
C_{21 p}(k), C_{22 p}(k) \\
C_{31 p}(k), C_{32 p}(k), C_{33 p}(k) \\
\vdots \\
C_{m 1 p}(k), C_{m 2 p}(k), \cdots, \dot{C}_{m m p}(k)
\end{array}\right)
$$

Since $\underline{A}_{p k}=\boldsymbol{C}_{p k} \underline{\zeta}_{p k}$,

$$
E\left(\underline{A}_{p k} \underline{A}_{p k^{T}}\right)=C_{p k} E\left(\zeta_{p k} \underline{\zeta}_{p k^{T}}\right) C_{p k^{T}}
$$

Since $E\left(\underline{A}_{p k} A_{p k}{ }^{T}\right)=\boldsymbol{K}_{p k}$ and

$E\left(\zeta_{p k} \zeta_{p k}{ }^{T}\right)=$ unit matrix,

$$
\boldsymbol{K}_{p k}=C_{p k} C_{p k}{ }^{T}
$$

Since eq. (12) is the same form of eq. (5) and since $\boldsymbol{K}_{p k}$ is a real symmetric matrix, $\boldsymbol{C}_{p k}$ can be similarly obtained. Thus, the element $a_{i p}(k)$ of $\underline{A}_{p k}$ can be calculated by

$$
a_{i p}(k)=\sum_{j=1}^{i} C_{i j p}(k) \zeta_{j p}(k)
$$

Since $b_{i p}(k)$ and $a_{i p}(k)$ are independent but identically distributed, the following random vector with zero mean

$$
\begin{array}{r}
\underline{B}_{p k}=\left\{b_{1 p}(k), b_{2 p}(k), \cdots, b_{i p}(k),\right. \\
\left.\cdots, b_{s p}(k), \cdots, b_{m p}(k)\right\}
\end{array}
$$

can be obtained independently from $A_{p k}$ in the similarmanner. 


\section{ERGODIC PROCESS}

In the following part, it will be proved briefly that the stationary Gaussian process, eq. (1) becomes ergodic when $N$ is infinity. For this purpose, assume eq. (1) as a sample function. It is easy to show that the temporal mean $\left\langle f_{i}(t)\right\rangle$ is zero;

$$
\left\langle f_{i}(t)\right\rangle=\lim _{T \rightarrow \infty} \frac{1}{T} \int_{0}^{T} f_{i}(t) d t=0
$$

The temporal autocorrelation function is

$$
\begin{aligned}
& \left\langle f_{i}(t) f_{i}(t+\tau)\right\rangle=\lim _{T \rightarrow \infty} \frac{1}{T} \int_{0}^{T} f_{i}(t) f_{i}(t+\tau) d t \\
& =\sum_{p=1}^{i} \sum_{q=1}^{i} \sum_{k=1}^{N} \frac{1}{2}\left\{a_{i p}(k) a_{i q}(k)+b_{i p}(k) b_{i q}(k)\right\} \\
& \quad \times \cos \left\{\omega_{k} \tau+\alpha_{i p}(k)-\alpha_{i q}(k)\right\} \quad \ldots . . .(16)
\end{aligned}
$$

The expectation of eq. (16) is therefore with eq. (7)

$$
\begin{aligned}
E & \left\{\left\langle f_{i}(t) f_{i}(t+\tau)\right\rangle\right\} \\
& =\sum_{p=1}^{i} \sum_{k=1}^{N} K_{i i p}(k) \cos \omega_{k} \tau=R_{i i}(\tau)
\end{aligned}
$$

This showns the mean of the temporal autocorrelation function is equal to the autocorrelation function in the ensemble sense. The variance of $\left\langle f_{i}(t) f_{i}(t+\tau)\right\rangle$ can be calculated as

$$
\begin{aligned}
\operatorname{var} & \left\{\left\langle f_{i}(t) f_{i}(t+\tau)\right\rangle\right\} \\
= & \frac{1}{2} \sum_{p=1}^{i} \sum_{k=1}^{N}\left[E\left\{a_{i p^{4}}(k)\right\}-E\left\{a_{i p^{2}}(k)\right\}^{2}\right] \\
& \times \cos ^{2} \omega_{k} \tau
\end{aligned}
$$

Thus,

$$
\begin{aligned}
\operatorname{var} & \left\{\left\langle f_{i}(t) f_{i}(t+\tau)\right\rangle\right\} \\
& \leqq \frac{1}{2} \sum_{p=1}^{i} \sum_{k=1}^{N}\left[E\left\{a_{i p^{4}}(k)\right\}-E\left\{a_{i p^{2}}(k)\right\}^{2}\right]
\end{aligned}
$$

Since $E\left\{a_{i p^{4}}(k)\right\}=3 E\left\{a_{i p^{2}}(k)\right\}^{2}$ for the Gaussian variable $a_{i p}(k)$ with zero mean, and since $E\left\{a_{i p^{2}}(k)\right\}=2\left|H_{i p}\left(\omega_{k}\right)\right|^{2}\left(\frac{\omega_{u}-\omega_{l}}{N}\right)$ from eqs. (3) and (4),

$$
\begin{aligned}
& \operatorname{var}\left\{\left\langle f_{i}(t) f_{i}(t+\tau)\right\rangle\right\} \\
& \quad \leqq \sum_{p=1}^{i} \sum_{k=1}^{N} 4\left|H_{i p}\left(\omega_{k}\right)\right|^{4}\left(\frac{\omega_{u}-\omega_{l}}{N}\right)^{2}
\end{aligned}
$$

Letting $H_{\max }=$ maximum of $\sum_{p=1}^{i} 4\left|H_{i p}\left(\omega_{k}\right)\right|^{4}$,

$$
\operatorname{var}\left\{\left\langle f_{i}(t) f_{i}(t+\tau)\right\rangle\right\} \leqq \frac{H_{\max }\left(\omega_{u}-\omega_{l}\right)^{2}}{N}
$$

Thus, $\lim _{N \rightarrow \infty} \operatorname{var}\left\{\left\langle f_{i}(t) f_{i}(t+\tau)\right\rangle\right\}=0$

Eqs. (15), (17) and (18) indicate that $f_{i}(t)$ becomes ergodic in the mean and autocorrelation function if $N$ is large in practice.

\section{APPLICATIONS}

\section{(1) Random Vibration of a Rectangular Plate}

Consider lateral vibration of a rectangular plate supported simply and subjected to spatially correlated lateral excitation. This problem was previously solved analytically by employing a perturbation method and formulated for the mean square response of the deflection ${ }^{6}$. However, only a special case of completely correlated spatial distribution of excitation was numerically solved since the multi-integrations involved in the formulation were not feasible if not impossible. Here the difficulty is avoided by simulation and the effect of degree of correlation of the excitation is considered.

The problem is governed by

$$
\begin{aligned}
& D\left(\frac{\partial^{4} w}{\partial \zeta^{4}}+2 \frac{a^{2}}{b^{2}} \frac{\partial^{4} w}{\partial \zeta^{2} \partial \eta^{2}}+\frac{a^{4}}{b^{4}} \frac{\partial^{4} w}{\partial \eta^{4}}\right) \\
& \quad+a^{4} \rho h \frac{\partial^{2} w}{\partial t^{2}}+a^{4} c \frac{\partial w}{\partial t}=a^{4} Q(\zeta, \eta, t) \ldots \ldots
\end{aligned}
$$

and the boundary conditions of no deflection and no moment at each edge of the plate.

Here, $w(\zeta, \eta, t)=$ lateral displacement

$$
\begin{aligned}
& D=\text { plate flexural rigidity }=E h^{3} / 12\left(1-\nu^{2}\right) \\
& h=\text { thickness of the plate } \\
& E=\text { Young's modulus } \\
& \nu=\text { Poisson's ratio } \\
& c=\text { coefficient of viscous damping } \\
& a=\text { width of the plate } \\
& b=\text { length of the plate } \\
& \rho=\text { mass density of the plate } \\
& \text { and } Q=\text { lateral excitation as a function of } \zeta, \eta
\end{aligned}
$$

Nondimensional coordinates $\zeta$ and $\eta$ are used by replacing $x$ and $y$ by $\zeta=x / a$ and $\eta=y / b$.

The following excitation force $Q(\zeta, \eta, t)$ is considered; $Q(\zeta, \eta, t)$ is formed of two parts,

$$
Q(\zeta, \eta, t)=Q_{0}(t)\{1+q(\zeta, \eta)\}
$$

$Q_{0}(t)$ is a stationary random process of time $t$ with zero mean and $q(\zeta, \eta)$ is also a stationary random process of $\zeta$ and $\eta$ with zero mean. $Q(\zeta, \eta, t)$ has the uniform spatial average intensity $Q_{0}(t)$ with variation $q(\zeta, \eta)$ over each location of the plate. $Q_{0}(t)$ and $q(\zeta, \eta)$ are assumed to be statistically independent. Since this is a case of a single random process, the following simple simulation is possible from eq. (1)

$$
Q_{0}(t) \cong \sum_{k=1}^{N}\left(a_{k} \cos \omega_{k} t+b_{k} \sin \omega_{k} t\right)
$$

where $a_{k}$ and $b_{k}$ are independent but with an identical Gaussian distribution with zero mean 
and with variance

$$
\sigma_{k}^{2}=2 S\left(\omega_{k}\right) \Delta \omega
$$

where $S\left(\omega_{k}\right)$ is the power spectral density function.

As to $q(\zeta, \eta)$ which is a two dimensional process, the following simulation is possible.

$$
\begin{aligned}
q(\zeta, \eta)= & \sum_{k_{1}=1}^{N} \sum_{k_{2}=1}^{N}\left\{a_{k_{1} k_{2}} \cos \left(\omega_{k_{1}} \zeta+\omega_{k_{2}} \eta\right)\right. \\
& \left.+b_{k_{1} k_{2}} \sin \left(\omega_{k_{1}} \zeta+\omega_{k_{2}} \eta\right)\right\}
\end{aligned}
$$

in which $a_{k_{1} k_{2}}$ and $b_{k_{1} k_{2}}$ are independent but identically distributed Gaussian random variables with zero mean and with variance

$$
\sigma_{k_{1} k_{2}}^{2}=S\left(\omega_{k_{1}}, \omega_{k_{2}}\right) \Delta \omega_{1} \Delta \omega_{2}
$$

in which $S\left(\omega_{k_{1}}, \omega_{k_{2}}\right)$ is the two dimensional spectral density function of $q(\zeta, \eta)$ and

$$
\Delta \omega_{i}=\frac{\omega_{u i}-\omega_{l i}}{N}, \quad \omega_{k_{i}}=\omega_{l i}+\left(k_{i}-\frac{1}{2}\right) \Delta \omega_{i}
$$

$\omega_{u i}$ and $\omega_{l i}$ are the upper and lower cutoff frequencies in the range $(-\infty, \infty)$. This simulation is valid since the expectation of eq. (23) is zero and the Wiener-Khintchine relations, $R\left(\tau_{1}, \tau_{2}\right)=$ $\int_{-\infty}^{\infty} \int_{-\infty}^{\infty} S\left(\omega_{1}, \omega_{2}\right) \cos \left(\omega_{1} \tau_{1}+\omega_{2} \tau_{2}\right) d \omega_{1} d \omega_{2}$ can be obtained from eqs. (23) and (24) when $N \rightarrow \infty$.

Following the usual procedure of analysis with the equations (21) and (23), the governing equation (19) can be solved as follows;

For the deflection as the steady state response,

$$
\begin{aligned}
w(\zeta, \eta, t)=\sum_{m=1}^{\infty} \sum_{n=1}^{\infty} \sum_{k=1}^{N} \sin m \pi \zeta \sin n \pi \eta\left|U_{m n}\left(\omega_{k}\right)\right| \\
\times\left[a_{k} \cos \left\{\omega_{k} t+\theta_{m n}\left(\omega_{k}\right)\right\}+b_{k} \sin \left\{\omega_{k} t+\theta_{m n}\left(\omega_{k}\right)\right\}\right]
\end{aligned}
$$

where

$$
\begin{aligned}
\left|U_{m n}\left(\omega_{k}\right)\right|=\frac{P_{m n}}{\rho h} \frac{1}{\sqrt{\left(\omega_{m m^{2}}-\omega_{k^{2}}\right)^{2}+c^{2} \omega_{k^{2}} / \rho^{2} h^{2}}} & \cdots \cdots(26) \\
P_{m n}= & \frac{4(1-\cos m \pi)(1-\cos n \pi)}{m n \pi^{2}} \\
& +4 \sum_{k_{1}=1}^{N} \sum_{k_{2}=1}^{N}\left\{a_{k_{1} k_{2}}\left(A_{m} B_{n}-C_{m} D_{n}\right)\right. \\
& \left.+b_{k_{1} k_{2}}\left(C_{m} B_{n}+D_{n} A_{m}\right)\right\} \\
A_{m}= & \frac{1-\cos \left(m \pi+\omega_{k_{1}}\right)}{2\left(m \pi+\omega_{k_{1}}\right)}+\frac{1-\cos \left(m \pi-\omega_{k_{1}}\right)}{\left(2 m \pi-\omega_{k_{1}}\right)} \\
B_{n}= & \frac{1-\cos \left(n \pi+\omega_{k_{2}}\right)}{2\left(n \pi+\omega_{k_{2}}\right)}+\frac{1-\cos \left(n \pi-\omega_{k_{2}}\right)}{2\left(n \pi-\omega_{k_{2}}\right)} \\
C_{m}= & \frac{\sin \left(m \pi-\omega_{k_{1}}\right)}{2\left(m \pi-\omega_{k_{1}}\right)}-\frac{\sin \left(m \pi+\omega_{k_{1}}\right)}{2\left(m \pi+\omega_{k_{1}}\right)}
\end{aligned}
$$

and

$$
D_{n}=\frac{\sin \left(n \pi-\omega_{k_{2}}\right)}{2\left(n \pi-\omega_{k_{2}}\right)}-\frac{\sin \left(n \pi+\omega_{k_{2}}\right)}{2\left(n \pi+\omega_{k_{2}}\right)}
$$$$
\omega_{m n^{2}}=\frac{D \pi^{4}}{a^{4} \rho h}\left(m^{2}+\frac{a^{2}}{b^{2}} n^{2}\right)^{2}
$$

$\omega_{m n^{2}}{ }^{2}$ is the square of the natural circular frequency of the undamped free vibration.

And,

$$
\theta_{m n}\left(\omega_{k}\right)=\tan ^{-1}\left\{-\frac{c \omega_{k}}{\rho h\left(\omega_{m n}^{2}-\omega_{k}^{2}\right)}\right\}
$$

The mean square response is

$$
\begin{aligned}
E & \left\{w^{2}(\zeta, \eta, t)\right\} \\
& =\sum_{m=1}^{\infty} \sum_{n=1}^{\infty} \sum_{p=1}^{\infty} \sum_{q=1}^{\infty} \sum_{k=1}^{N} \sin m \pi \zeta \sin n \pi \eta \\
& \times \sin p \pi \zeta \sin q \pi \eta E\left\{\left|U_{m n}\left(\omega_{k}\right)\right|\left|U_{p q}\left(\omega_{k}\right)\right|\right\} \sigma_{k}^{2} \\
& \times \cos \left\{\theta_{m n}\left(\omega_{k}\right)-\theta_{p q}\left(\omega_{k}\right)\right\}
\end{aligned}
$$

where

$$
\begin{aligned}
& E\left\{\left|U_{m n}\left(\omega_{k}\right)\right|\left|U_{p q}\left(\omega_{k}\right)\right|\right\} \\
& =\frac{E\left(P_{m n} P_{p q}\right)}{\rho^{2} h^{2} \sqrt{\left(\omega_{m n^{2}}-\omega_{k^{2}}\right)^{2}+c^{2} \omega_{k}^{2} / \rho^{2} h^{2}}} \\
& \times \frac{1}{\sqrt{\left(\omega_{p q^{2}}-\omega_{k}^{2}\right)^{2}+c^{2} \omega_{k}^{2} / \rho^{2} h^{2}}}
\end{aligned}
$$

$$
\begin{aligned}
& E\left(P_{m n} P_{p q}\right)=16(1-\cos m \pi)(1-\cos n \pi) \\
& \quad \times(1-\cos p \pi)(1-\cos q \pi) / m n p q \pi^{4} \\
& +16 \sum_{k_{1}=1}^{N} \sum_{k_{2}=1}^{N} \sigma_{k_{1} k_{2}}^{2} \\
& \quad \times\left\{\left(A_{m} B_{n}-C_{m} D_{n}\right)\left(A_{p} B_{q}-C_{p} D_{q}\right)\right. \\
& \left.+\left(C_{m} B_{n}+D_{n} A_{m}\right)\left(C_{p} B_{q}+D_{q} A_{p}\right)\right\} \ldots
\end{aligned}
$$

It is noted that the mean square response eq. (31) does not require generation of random variables and is given in the deterministic form.

Consider the case when $Q_{0}(t)$ is taken to be a low-pass white noise with $S(\omega)=S_{0}$ (constant) for $-B \leq \omega \leq B$. Thus the autocorrelation function is $R(\tau)=\sigma_{Q_{0}}{ }^{2} \frac{\sin B \tau}{B \tau}$ where $\sigma_{Q_{0}}{ }^{2}=$ variance of $Q_{0}(t)$. For large $B, Q_{0}(t)$ becomes effectively an ideal white noise. The power spectral density function of $q(\zeta, \eta)$ is also $S\left(\omega_{1}, \omega_{2}\right)=c$ for $-u \leq \omega_{1}, \omega_{2} \leq u$. The autocorrelation function is therefore $R\left(\tau_{1}, \tau_{2}\right)$ $=\sigma_{q}^{2}\left(\frac{\sin u \tau_{1}}{u \tau_{1}}\right)\left(\frac{\sin u \tau_{2}}{u \tau_{2}}\right)$ where $\sigma_{q}^{2}=4 c u^{2}=$ variance of $q(\zeta, \eta)$.

It is noted that the bound $u$ can serve as a parameter of correlation since when $u \rightarrow 0, R\left(\tau_{1}, \tau_{2}\right)$ $=\sigma_{q}{ }^{2}$, uniform correlation and when $u \rightarrow \infty$, $R\left(\tau_{1}, \tau_{2}\right)=\sigma_{q}{ }^{2} \delta\left(\tau_{1}\right) \delta\left(\tau_{2}\right)$, independent for $\tau_{1}=\tau_{2} \neq 0$.

For the uniform correlation of $q(\zeta, \eta)$, the mean square response eq. (31) is calculated by using 


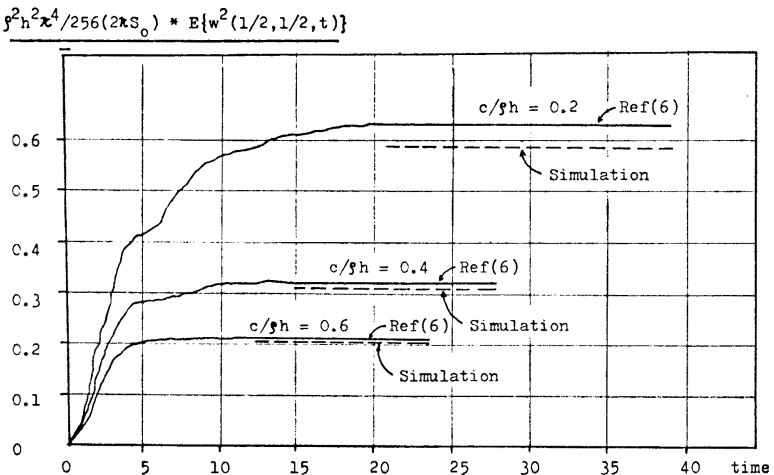

Fig. 1 Mean Square Response of Deflection.

$$
R_{i j}(\tau)=\varepsilon_{i j} \sigma_{i} \sigma_{j} \exp (-\kappa|\tau|) ; \quad i, j=1,2
$$

where $\sigma_{i}$ and $\sigma_{j}$ are the standard deviations of $f_{i}(t)$ and $f_{j}(t)$ respectively. $\varepsilon_{i j}=\varepsilon$ for $i \neq j$ and 1 for $i=j . \quad \varepsilon$ is a constant value between zero and one. $\kappa$ is a positive constant governing the shape of the correlation. Note that the value of $\varepsilon$ serves as a correlation parameter since $\varepsilon=0$ and $\varepsilon=$ 1.0 indicate respectively independence and complete correlation between $f_{i}(t)$ and $f_{j}(t)$. By the Wiener-Khintchine relation, the real and symmetric cross-spectral density function is given from eq. (34)

$$
S_{i j}(\omega)=\frac{\varepsilon_{i j} \sigma_{i} \sigma_{j} \kappa}{\pi\left(\kappa^{2}+\omega^{2}\right)}
$$

the first five modes and with $D \pi^{4} / a^{4} \rho h=0.1, B=$ 100 and $N=500$. The Fig. 1 shows the mean square deflection of the middle point of a square plate $(a / b=1)$ together with the transient nonstationary responses by reference ${ }^{6)}$. The difference of the both results decreases when the damping becomes large. The response values in reality should be between those of independent $q$ and completely correlated $q$ since actual load must be somehow correlated along spatial coordinates. The Table 1 shows that for large $\sigma_{q}{ }^{2}$, the effect of correlation becomes significant.

Table 1 Mean Square Response of Deflection.

\begin{tabular}{c|c|cc}
\hline \multirow{2}{*}{$\mu=c / \rho h$} & \multicolumn{3}{|c}{ Mean Square Response } \\
\cline { 2 - 4 } & $\begin{array}{c}\text { Independent } \\
q\end{array}$ & Completely Correlated $q$ \\
\hline & & $\sigma_{q}{ }^{2}=0.01$ & $\sigma_{q}{ }^{2}=0.05$ \\
0.2 & 5.728 & 5.785 & 6.014 \\
0.4 & 3.038 & 3.068 & 3.189 \\
0.6 & 2.020 & 2.040 & 2.121 \\
\hline
\end{tabular}

\section{(2) Simple Beam with a Set of Correlated Random Loadings}

Consider lateral vibration of a simple beam when it is subjected to a set of two correlated random loadings which are stationary Gaussian with zero mean. The beam is illustrated in Fig. 2. The cross-correlation function of $f_{i}(t)$ and $f_{j}(t)$ is taken as

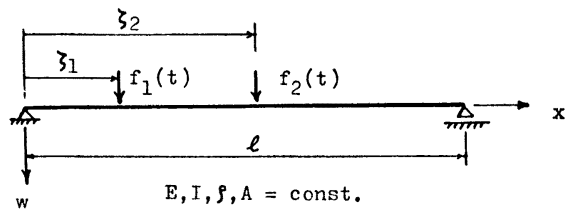

Fig. 2 A Simple Beam.
Using the foregoing simulation method eq. (1), the deflection of the middle point $(x=l / 2)$ for the given loadings at $\zeta_{1}=l / 3$ and $\zeta_{2}=2 l / 3$ is simulated where the following values are assigned; $\sigma_{1}=\sigma_{2}$ $=3.0, \kappa=2.0$ and $N=500$. The value $\varepsilon$ was varied for the purpose of the examination of the effect of correlation of $f_{1}(t)$ and $f_{2}(t)$ upon the response process. The upper cutoff frequency $\omega_{u}=4 \pi$ radian/sec is employed. Thus, the numerical evaluation of sample histories is performed with every time interval $\Delta t=0.25 \mathrm{sec}$. For the system

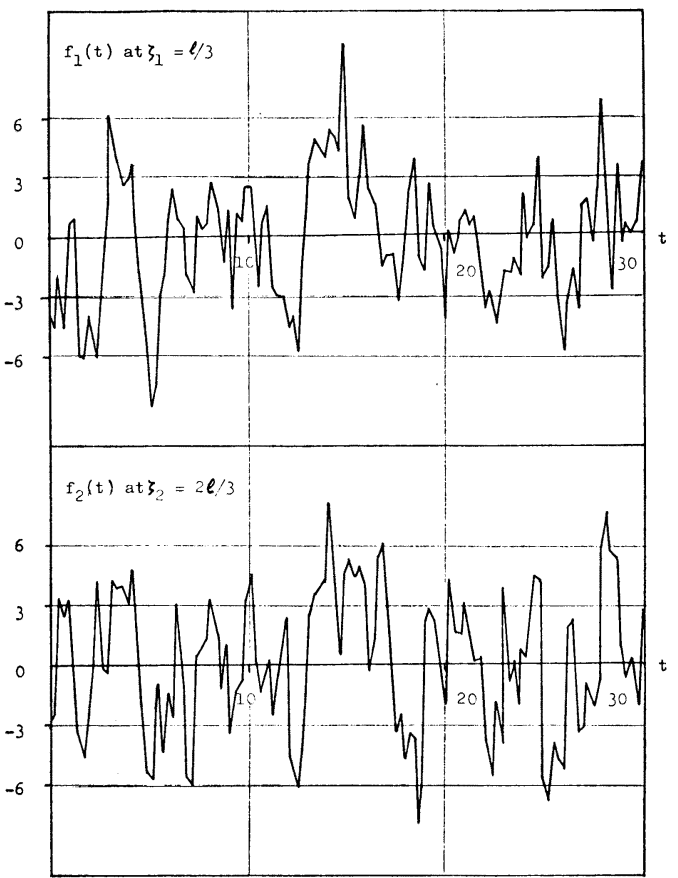

Fig. 3 A Set of Random Loadings (the correlation, $\varepsilon=0.5$ ). 
property, $\quad \pi^{2} / l^{2} \sqrt{E I / \rho A}=5.0$ and $c / \rho A=3.0$ are assumed. This is a case of low damping. The dimension of data is not specified since this is a demonstration.

Fig. 3 shows a set of random loadings for the time duration 30 seconds with the correlation $\varepsilon=$ 0.5. As expected, it shows fairly wide band processes. The response process of the middle point deflection is narrowed as indicated in Fig. 4. The mean square deflection vs. the correlation $\varepsilon$ is shown in Fig. 5. The mean square response varies dependent upon the value $\varepsilon$. The difference between the two extreme cases (perfect

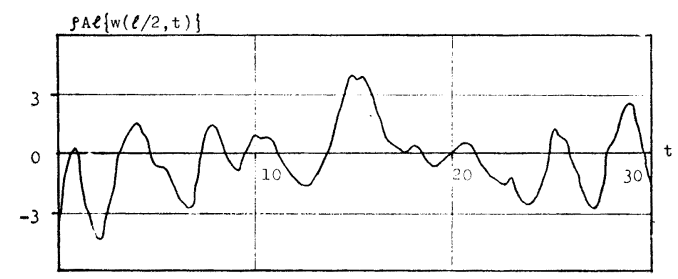

Fig. 4 Deflection at the Middle Point.

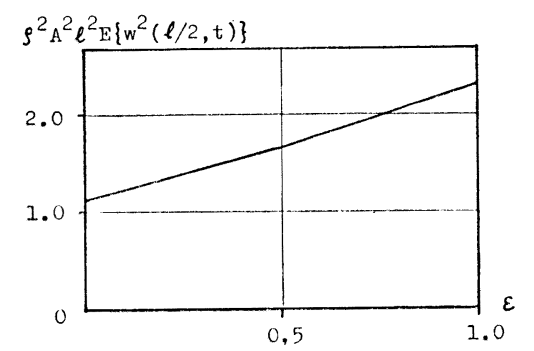

Fig. 5 Mean Square Response of Deflection. independence and perfect correlation of the set of loadings) is quite significant as seen in the figure.

\section{(3) Streamwise Strong Wind Turbulence}

Consider as a final example the simulation of two correlated longitudinal turbulence components at two fixed locations $A$ and $B$. The direction of the separation distance $A B$ is normal to the streamwise direction in the horizontal plane. Under strong wind conditions, the mixing action of the mechanical turbulence tends to reduce the atmosphere to a state of neutral stability. Consequently the turbulence near the ground is only due to frictional effects and will vary only significantly with the surface drag and height above the ground. If this is the case, the turbulence can be described as a stationary Gaussian process with zero mean. Simulated are the two components of turbulence with Davenport's empirical strong wind spectrum ${ }^{4}$

$$
S(\omega)=\frac{2 \kappa \bar{U}_{1}^{2}}{\omega} \frac{x^{2}}{\left(1+x^{2}\right)^{4 / 3}} \quad(\text { two sided })
$$

where $x=\frac{600 \omega}{\pi \bar{U}_{1}}$

and with the coherence function for the longitudinal turbulence components of the separation distance $\Delta y^{2)}$,

$$
\gamma(\omega)=\exp \left(-\frac{\kappa^{\prime} d y \omega / 2 \pi}{\bar{U}_{1}}\right) .
$$

$\bar{U}_{1}$ is the reference mean velocity near the ground at an elevation of 10 meters. $\kappa$ is the drag coefficient dependent on the surface roughness. $\kappa^{\prime}$

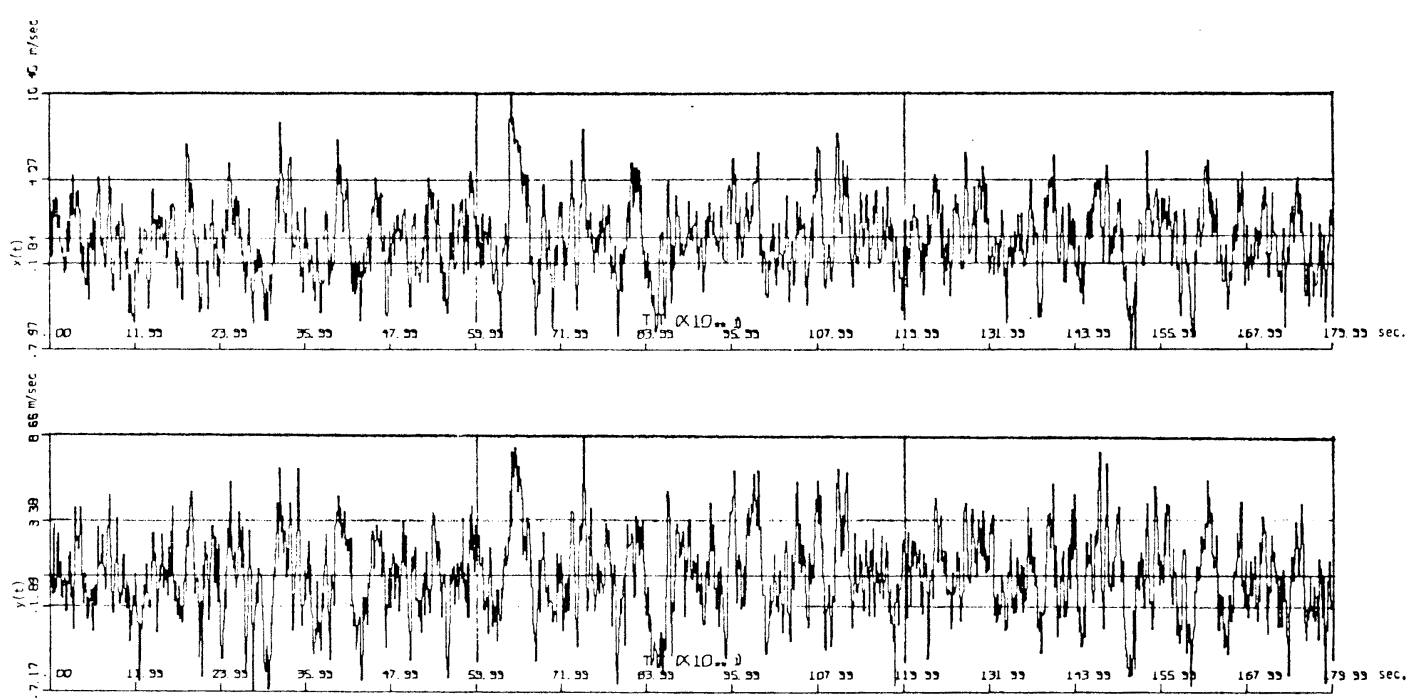

Fig. 6 A Set of Streamwise Turbulence. 
is a dimensionless coefficient between 20 and 25 . The quadrature spectrum is assumed to be zero since it is expected that the maximum correlation between the two components occurs at a zero lag.

Fig. 6 shows two sets of correlated streamwise turbulence components $x(t)$ and $y(t)$ when $N=600$, $\omega_{l}=0.00377 \mathrm{rad} / \mathrm{sec}$ and $\omega_{u}=3.14 \mathrm{rad} / \mathrm{sec}$. The simulated turbulence data were evaluated at $\Delta t=$ $1.0 \mathrm{sec}$ time interval. $\bar{U}_{1}=16.5 \mathrm{~m} / \mathrm{sec}, \quad \kappa=0.005$ and $\Delta y=5$ meters are used. Fig. 7 shows the hystograms of the amplitude of the simulated processes and are approximately Gaussian in na-
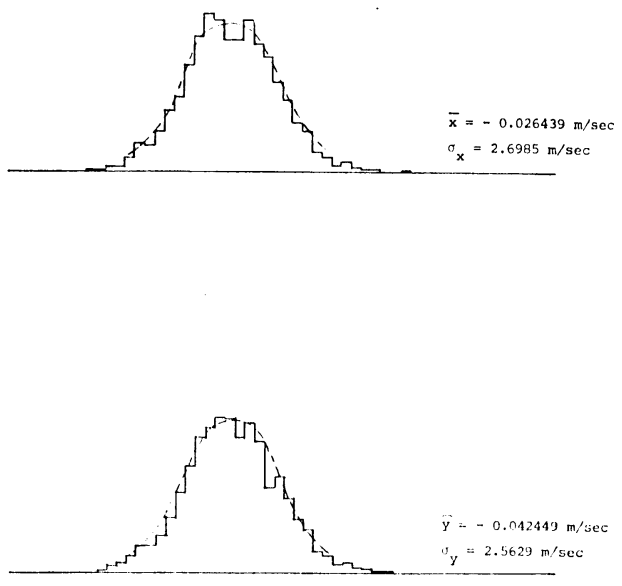

Fig. 7 Hystograms of Amplitude.

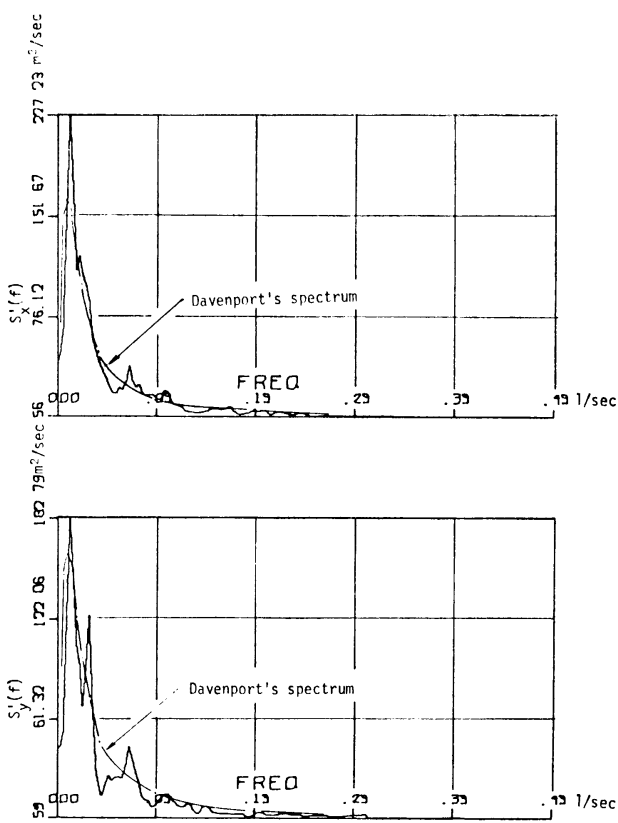

Fig. 8 Calculated Power Spectrum. ture. However, it shows a non zero mean value which can be attributed to the sample length. Fig. 8 shows the calculated spectral density function and Davenport's emperical spectrum from which the simulation was obtained. Fig. 9 shows the calculated co-spectral and quadrature spectral density functions. Because of the relatively small sample length (total 30 minutes) of the process, one may expect non-zero fluctuation for the quadrature spectrum at low frequencies.

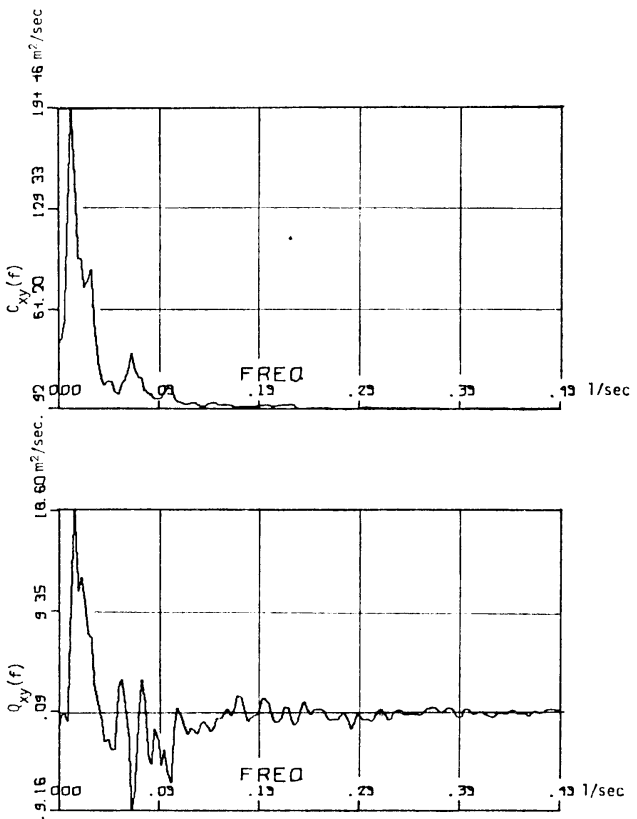

Fig. 9 Calculated Co-Spectrum and Quadrature Spectrum.

\section{CONCLUSIONS}

A method of simulation of a set of $m$ multicorrelated stationary Gaussian processes with zero mean is developed by a trigonometric series representation with Gaussianly distributed coefficients. This model becomes ergodic if the total number of the summation $N$ in the model is infinity. Examples are also presented for the possible applications in the random vibration problems.

\section{ACKNOWLEDGEMENTS}

The author apprecates the department of Engineering Mechanics, Virginia Polytechnic Institute and State University where the theoretical simulation method was studied. The applications of this method were investigated in Civil Engineer- 
ing Department, Musashi Institute of Technology since October, 1971. The encouragement and advice from Dr. T. Nishiwaki are very much appreciated.

\section{REFERENCES}

1) Amin, M. and Ang, A. H.-S.: “Nonstationary Stochastic Model of Earthquake Motions", Journal of EM Div., ASCE, Vol. 94, 1968.

2) Ang, A. H.-S. and Amin, M.: "Probabilistic Structural Mechanics and Engineering", Notes of NSF Summer Institute, Univ. of Illinois, June, 1970.

3) Borgman, L. E.: "Ocean Wave Simulation for Engineering Design", Journal of Waterways and Harbors Div., ASCE, No. WW4, Nov., 1969.

4) Davenport, A. G.: The Spectrum of Horizontal Gustiness near the Ground in High Winds", Journal of Royal Meteorological Soc., Vol. 87, No. 372, April, 1961.

5) Goto, H. et al.: "Artificial Earthquake Waves by Computer", Proc. of Japan Earthquake Eng. Symposium, 1966.

6) Hoshiya, M. and Shah, H. C.: “Dynamics and Eigenvalue Analysis of a Rectangular Plate with Stochastic Properties", Proc. of
Japan Society of Civil Engineers, No. 187, March, 1971.

7) Housner, G. W. and Jennings, P. C.: “Generation of Artificial Earthquakes", Journal of EM Div., ASCE, Vol. 90, Feb., 1964.

8) IBM System $/ 360$ Scientific Subroutine Package, Form No. H20-0205-3, Subroutine RAN. DU, 1969.

9) Liu, S. C.: “Dynamics of Correlated Random Pulse Trains", Journal of EM Div. ASCE, Vol. 96, Aug., 1970.

10) Rice, S. O.: "Mathematical Analysis of Random Noise", in Selected Papers on Noise and Stochastic Processes, edited by Wax N., Dover, N. Y., 1955.

11) Shreider, Yu. A.: “The Monte Carlo Method", Pergamon Press, 1966, p. 328.

12) Shinozuka, M.: "Simulation of Multivariate and Multidimensional Random Processes", Journal of Accoustical Soc. of Am., Vol. 49, No. 1, Jan., 1971.

13) Shinozuka, M. and Jan, C. M.: "Simulation of Multivariate and Multidimensional Processes II", Tech. Report No. 12, Columbia Univ. N. Y., April, 1971.

(Received Dec. 6, 1971) 